\title{
Correspondence
}

\section{Ease of use of the Airway Scope vs the Bullard laryngoscope: a manikin study}

To the Editor:

Rigid fibreoptic laryngoscopes provide a non-line-ofsight view of the airway. Tracheal intubation using a rigid laryngoscope is one of the alternative methods used for rescue intubation when physicians encounter a difficult airway. However, the device is not widely used, in part because it is perceived as being difficult to use. " The Airway Scope (AWS; Pentax Corp., Tokyo, Japan) is a new intubation device that allows visualization of the glottis without requiring alignment of the oral, pharyngeal and tracheal axes. ${ }^{2} \mathrm{We}$ recently compared the ease of intubation with the AWS compared to the Bullard laryngoscope (BLS; Circon ACMI, Stamford, CT, USA), as used by novice personnel with a manikin model.

Nine anesthesia residents, with no prior experience using either the AWS or the BLS, were given a standardized demonstration of both devices. Each participant was then allowed to practice intubations using each device on an Intubation Trainer (Laerdal Medical, Tokyo, Japan). At each stage, all participants could successfully perform tracheal intubation with both devices. Each participant performed tracheal intubation using a 7-mm cuffed tracheal tube on a SimMan manikin (Laerdal Medical, Tokyo, Japan) in the following laryngoscopy scenarios: 1) normal airway with neutral head and neck position, 2) difficult laryngoscopy scenario with rigid cervical spine, and 3) difficult laryngoscopy scenario with tongue edema. The sequence in which each participant used the AWS and the BLS was randomly assigned using numbers drawn from a random numbers table. Each intubator repeated the examination protocol three times, and the sequence of the devices was alternated at the second airway instrumentation. The duration of each tracheal intubation attempt was defined as the time taken from insertion until removal of the blade from between the teeth. Participants scored the ease of use of each device on a visual analogue scale (VAS; from $0=$ extremely easy to $100 \mathrm{~mm}=$ extremely difficult $)$. The quality of the laryngeal view and intubation status using each device was also scored on a VAS scale $(0$ $=$ extremely good to $100 \mathrm{~mm}=$ extremely poor $)$. The
TABLE Time (sec) required for intubation of the trachea using the Airway Scope (AWS) and Bullard laryngoscope (BLS) in three different scenarios using SimMan ${ }^{\circledR}$

\begin{tabular}{lll}
\hline & $A W S$ & \multicolumn{1}{l}{$B L S$} \\
\hline Neutral head and neck & $8.3 \pm 3.0 \ddagger$ & $16.6 \pm 11.4$ \\
Rigid cervical spine & $9.8 \pm 2.4 \dagger$ & $23.6 \pm 21.8$ \\
Tongue edema & $18.0 \pm 17.0^{*}$ & $36.1 \pm 35.9$ \\
\hline Data are mean $\pm S D .{ }^{*} P<0.05 ; \dagger P<0.01 ; \ddagger P<0.001$ ps BLS.
\end{tabular}

two groups were compared using Student's $t$ test, and data are presented as mean \pm SD. A $P$ value $<0.05$ was considered statistically significant.

For each scenario, the duration of intubation was significantly shorter with the AWS compared to the BLS (Table) and the degree of intubation difficulty was less with the AWS compared to the BLS $(18 \pm 14$ vs $72 \pm$ $13, P<0.0001)$. The quality of the laryngeal view based on VAS scores was also better with the AWS compared to the BLS $(15 \pm 16$ vs $74 \pm 25, P<0.0001)$

Despite their lack of experience and unfamiliarity with the AWS, anesthesia residents who were novice to the techniques performed tracheal intubation more quickly with the AWS, and expressed a greater preference for the AWS compared to the BLS. The high resolution charge-coupled camera and the target signal shown on the monitor were helpful for handling the AWS. These preliminary data suggest that the AWS may be a useful device even with novice users, particularly when intubation requires a non-line-ofsight view of the airway for difficult intubations. These preliminary findings are limited by the operator's experience and the interpretation from a manikin model. Further studies are warranted to determine the potential role of the AWS for airway management in clinical settings.

Yoshihiro Hirabayashi MD

Jichi Medical University, Tochigi, Japan

E-mail: yhira@jichi.ac.jp

Support was provided solely from institutional and/or departmental sources. The author has no affiliation with any manufacturer of any devices described in the manuscript.

Accepted for publication January 11, 2007. 


\section{References}

1 Wong DT, Lai K, Chung FF, Ho RY. Cannot intubatecannot ventilate and difficult intubation strategies: results of a Canadian national survey. Anesth Analg 2005; 100: 1439-46.

2 Koyama J, Aoyama T, Kusano $\Upsilon$, et al. Description and first clinical application of AirWay Scope for tracheal intubation. J Neurosurg Anesthesiol 2006; 18: 24750 .

\section{Trachlight $\AA$ - More practical solutions to commonly encountered problems}

To the Editor:

Over the past few decades, light-guided intubation using the principle of transillumination has proven to be an effective, safe and simple technique. A lighted stylet uses the principle of transillumination of the soft tissues of the anterior neck to guide the tip of the endotracheal tube into the trachea. This technique takes advantage of the anterior or more superficial location of the trachea in relation to the esophagus.

Several tips have been suggested in the literature for improving the success of Trachlight ${ }^{\circledR}$-guided intubation. These include lifting the tongue with the thumb of the nondominant hand or having an assistant pull the tongue while the intubator continues to provide a jaw thrust, lubricating the wand and the stylet, ${ }^{1}$ dimming operating room lights, using smaller size endotracheal tubes, inserting the wand side-on, or providing at least a $90^{\circ}$ bend to the wand. ${ }^{2}$ Others have suggested gauging the appropriate "bent length" by measuring the distance from the thyroid prominence to the angle of the mandible. ${ }^{3}$ Patients with buck teeth may benefit from the addition of another bend to the wand at the level of the buck teeth. ${ }^{4}$

Following repeated use, the internal rigid stylet sometimes assumes a "snake-like" bend that poses difficulty in retraction of the wand. In such situations, it has been suggested that the stylet be straightened, if possible, before reuse, failing which it needs to be disposed. ${ }^{5}$ We have also encountered a similar situation leading to difficulty in withdrawing the wand along with the rigid internal stylet after successfully negotiating the endotracheal tube-Trachlight ${ }^{\circledR}$ assembly into the trachea. We have noticed that the snake-like bend of the stylet poses a problem when it crosses the endotracheal tube connector, which happens to be not only the narrowest, but also the most rigid portion of the endotracheal tube-connector assembly. We have successfully overcome "hold up" at this level by separating the endotracheal tube connector from the endotracheal tube prior to withdrawal of the wand-stylet assembly. The distal $90^{\circ}$ bent portion of the wand-stylet assembly is the other point at which difficulty is encountered during withdrawal of the stylet, especially in the pediatric age group due to the small size of the tube and its connector. Our suggestion provides a solution to this problem also. We therefore recommend that the connector be routinely separated from the endotracheal tube to facilitate smooth removal of the stylet and possibly prolong the life of the stylet. We have applied this technique of removal of the endotracheal tube connector to aid in Trachlight ${ }^{\circledR}$-guided oral intubation using the Ring Adair Elwyn (Mallinckrodt Medical, Athlone, Ireland) tube also.

In obese individuals, the midline tissues of the neck may be obscured by folds of fat arising either from a double-chin above, or from the anterior chest wall below, posing difficulty in appreciation of the circumscribed glow in front of the neck. Dimming the operating room lights and placing a support under the shoulder to extend the neck often improves success of Trachlight ${ }^{\circledR}$-guided intubation in obese patients. We have found that having an assistant retract the fold of fatty tissue down and away from the neck so as to avoid formation of skin folds over the neck helps in shortening the time to obtain the classical well-circumscribed midline glow.

Since its introduction in 1959, the lightwand has proven its utility in several clinical situations. Our experience gleaned from the use of the Trachlight ${ }^{\circledR}$ for more than 350 intubations has prompted us to share some of the practical solutions that we have used to overcome problems that we have commonly encountered during its use.

Goneppanavar Umesh MD

George Mathew MBBS

Venkateswaran Ramkumar MD

Kasturba Medical College, Manipal, India

E-mail: drumeshg@yahoo.co.in

Accepted for publication January 15, 2007.

\section{References}

1 Hung OR, Pytka S, Morris I, et al. Clinical trial of a new lightwand device (Trachlight) to intubate the trachea. Anesthesiology 1995; 83: 509-14.

2 Djordjevic D. Trachlight ${ }^{\mathrm{TM}}$ - Learning tips. Can J Anesth 1999; 46: 615.

3 Chen TH, Tsai SK, Lin CJ, Lu CW, Tsai TP, Sun WZ. Does the suggested lightwand bent length fit every patient? The relation between bent length and patient's thyroid prominence-to-mandibular angle distance. 